\title{
TILINGS OF NORMED SPACES
}

\author{
CARLO ALBERTO DE BERNARDI AND LIBOR VESELÝ
}

\begin{abstract}
By a tiling of a topological linear space $X$ we mean a covering of $X$ by at least two closed convex sets, called tiles, whose nonempty interiors are pairwise disjoint. Study of tilings of infinite-dimensional spaces initiated in 1980's with pioneer papers by V. Klee. We prove some general properties of tilings of locally convex spaces, and then apply these results to study existence of tilings of normed and Banach spaces by tiles possessing certain smoothness or rotundity properties. For a Banach space $X$, our main results are the following.

(i) $X$ admits no tiling by Fréchet smooth bounded tiles.

(ii) If $X$ is locally uniformly rotund (LUR), it does not admit any tiling by balls.

(iii) On the other hand, some $\ell_{1}(\Gamma)$ spaces, $\Gamma$ uncountable, do admit a tiling by pairwise disjoint LUR bounded tiles.
\end{abstract}

\section{INTRODUCTION}

Let $X$ be a real topological vector space (t.v.s.). A set $B \subset X$ will be called a body if it is closed, convex and has nonempty interior. A tiling of $X$ is a covering of $X$ by at least two bodies whose interiors are pairwise disjoint. Members of a tiling are called tiles.

While there exists a wide theory of tilings (and their analogues with not necessarily convex "tiles") of finite dimensional spaces, especially the plane, the infinite dimensional theory is still much less developed, though the first "infinite dimensional" results appeared already in 1980's. The present paper is devoted to the question about existence, in infinite dimensional normed or Banach spaces, of tilings having some particular properties. Let us briefly recall main known results.

It is easy to see that some tilings always exist in any locally convex t.v.s. $X$. Indeed, if $f$ is a nonzero continuous linear functional on $X$ and $\mathcal{T}$ is a tiling of $\mathbb{R}$ then the family $\mathcal{B}=\left\{f^{-1}(T): T \in \mathcal{T}\right\}$ is a tiling of $X$. Each tiling of this form will be called a trivial tiling.

2010 Mathematics Subject Classification. Primary 46B20 ; Secondary 52A99, 46A45.

Key words and phrases. Tiling of normed space, Fréchet smooth body, locally uniformly rotund body, $\ell_{1}(\Gamma)$-space.

Research supported in part by the Ministero dell'Istruzione, dell'Università e della Ricerca of Italy and by GNAMPA-INdAM. 
Notice that no countable tiling can be disjoint (i.e., consisting of pairwise disjoint tiles) since otherwise a line in $X$ would admit a disjoint covering by at most countably many and at least two nonempty closed convex sets, which is known to be impossible (see Fact 3.2 or [3, Theorem 6.1.27]). It follows that a separable normed space does not admit any disjoint tiling. On the other hand, some (nonseparable) $\ell_{1}(\Gamma)$ does admit a disjoint tiling by balls of the same positive radius (see V. Klee [8]).

Tilings by bounded (convex) tiles always exist in normed spaces. In 1997, V.P. Fonf, A. Pezzotta and C. Zanco [6] proved that every normed space $X$ admits a tiling by bounded bodies each of which contains a ball of a certain fixed positive radius. Subsequently, A. Marchese and C. Zanco [12] showed that each normed space $X$ admits a tiling by bounded bodies such that each point of $X$ belongs to at most two of them. Since this tiling is "point-finite", a natural question arises: can such a tiling be always taken locally finite, in the sense that each point of $X$ has a neighborhood intersecting only finitely many tiles? An answer is contained in a paper by Fonf [5] who showed that $a$ separable Banach space admits a locally finite tiling by bounded bodies if and only if $X$ is isomorphically polyhedral. For nonseparable Banach spaces $X$ it is only known that if $X$ contains an infinite dimensional closed subspace non containing $c_{0}$ then $X$ does not admit any locally finite tiling by bounded bodies; this follows from a generalization, due to Fonf and Zanco [7], of a theorem of Corson (1961).

A tiling $\mathcal{B}$ is said to be equi-bounded below and above if there exist two constants $0<r<R<\infty$ such that each member of $\mathcal{B}$ contains a ball of radius $r$ and is contained in a ball of radius $R$. Already in 1986, V. Klee [9] showed that some nonseparable Hilbert spaces admit tilings that are equi-bounded below and above. The case of the separable (infinite dimensional) Hilbert space was settled only in 2010 by D. Preiss [13] in the positive: $\ell_{2}$ admits a tiling which is equi-bounded below and above. An interesting open problem is: which Banach spaces admit such tilings?

Our interest is devoted to the question of existence of (nontrivial) tilings by bodies that have a kind of smoothness or rotundity properties. The first such result, due to Klee and Tricot [11], affirms that a tiling of a Banach space by bounded tiles each of which is either smooth or rotund has to be uncountable. In particular, a separable Banach space admits no tiling by smooth or rotund bounded bodies. The only related known fact for nonseparable Banach spaces follows from results in [10] and says: if $X$ is a uniformly rotund or uniformly smooth Banach space then it does not admit any tiling by balls which would be either disjoint or bounded below (i.e., having radii bounded away from zero).

In the present paper, after proving some general results on tilings in locally convex t.v.s. (Section 3), we show that Banach spaces do not admit nontrivial tilings by Fréchet smooth bodies, and normed spaces do not admit disjoint 
tilings by Fréchet smooth bodies (see Theorem 4.2 and Corollary 4.3). Concerning existence of tilings by locally uniformly rotund (LUR) bodies, we show on the one hand that a LUR Banach space does not admit any tiling by balls, and a LUR normed space does not admit any disjoint tiling by balls; on the other hand, some nonseparable $\ell_{1}(\Gamma)$ admits a disjoint tiling by bounded centrally symmetric LUR bodies (see Section 5). This last construction is based on the above mentioned Klee's construction in [8]. We also show that some geometric conditions, concerning a single point of the unit sphere of a Banach space, imply nonexistence of tilings by balls whose radii are bounded away from zero; see Subsection 4.3.

\section{Notations AND PRELIMINARIES}

Throughout this paper, all normed, Banach or topological vector spaces are real and at least two-dimensional.

Let $X$ be a normed space with dual $X^{*}$. By $B_{X}$ and $S_{X}$ we denote the closed unit ball and the unit sphere of $X$, respectively. The polar set of a set $A \subset X$ is the set $A^{\circ}=\left\{f \in X^{*}: f(a) \leq 1\right.$ for each $\left.a \in A\right\}$.

Let $B \subset X$ be a body, that is, a closed convex set with nonempty interior. A point $x \in \partial B$ is a smooth point of $B$ if the set

$$
D_{B}(x)=\left\{f \in S_{X^{*}}: f(x)=\sup f(B)\right\}
$$

is a singleton. If $c \in \operatorname{int} B, B_{0}=B-c$ and $x_{0}=x-c$, it is standard to see (and well-known for balls) that $x$ is a smooth point of $B$ if and only if the Minkowski functional $p_{B_{0}}$ of $B_{0}$ is Gâteaux differentiable at $x_{0}$. We say that $x$ is a Fréchet smooth point of $B$ if $p_{B_{0}}$ is Fréchet differentiable at $x_{0}$. (It is standard to see that this definition does not depend on the choice of $c$.)

We say that $x \in \partial B$ is a $L U R$ (locally uniformly rotund) point of $B$ if for each $\varepsilon>0$ there exists $\delta>0$ such that if $y \in \partial B$ and $\operatorname{dist}(\partial B,(x+y) / 2)<\delta$ then $\|x-y\|<\varepsilon$. If $B=B_{X}$, this definition coincides with the standard definition of local uniform rotundity of the norm at $x$.

We say that a body $B$ is smooth, Fréchet smooth, or $L U R$ if each boundary point of $B$ is a smooth, Fréchet smooth, or LUR point of $B$, respectively.

For $f \in S_{X^{*}}$ and $\alpha \in[0,1)$, we consider the closed convex cone

$$
\mathrm{C}(\alpha, f)=\{x \in X: f(x) \geq \alpha\|x\|\} .
$$

Notice that $\mathrm{C}(\alpha, f)$ has nonempty interior.

Observation 2.1. Let $B \subset X$ be a body, $x \in \partial B$. If $x$ is a Fréchet smooth point of $B$ then there exists $f \in S_{X^{*}}$ with the following property: for each $\alpha>0$ there exists $\varepsilon>0$ such that

$$
[x-\mathrm{C}(\alpha, f)] \cap\left[x+\varepsilon B_{X}\right] \subset B .
$$

(Also the converse is true, but we shall need only the above implication.) 
Proof. Assume without any loss of generality that $0 \in \operatorname{int} B$. Let $g$ be the Fréchet derivative of $p_{B}$ at $x$, and $f:=\frac{g}{\|g\|}$. Then $p_{B}(x)=1$ and $\lim _{\|h\| \rightarrow 0} \frac{p_{B}(x+h)-p_{B}(x)-g(h)}{\|h\|}=0$. Consequently, for each $\alpha>0$ there exists $\varepsilon>0$ such that $p_{B}(x+h)-1-\|g\| f(h) \leq \alpha\|g\|\|h\|$ whenever $h \in \varepsilon B_{X}$. Thus, for $h \in[-\mathrm{C}(\alpha, f)] \cap \varepsilon B_{X}$, we obtain $p_{B}(x+h) \leq 1+\|g\| f(h)+\alpha\|g\|\|h\| \leq 1$, and hence $x+h \in B$. This completes the proof.

Definition 2.2. Let $B \subset X$ be a body, $x \in \partial B$ and $\varepsilon>0$. We say that $x$ is an $\varepsilon$-cone smooth point of $B$ if there exists $f \in S_{X^{*}}$ such that

$$
\left[x-\mathrm{C}\left(\frac{1}{7}, f\right)\right] \cap\left[x+\varepsilon B_{X}\right] \subset B,
$$

that is, (1) holds for $\alpha=1 / 7$. We denote by $\varepsilon$-CS $(B)$ the set of all $\varepsilon$-cone smooth points of $B$. We say that $x \in \partial B$ is a cone smooth point of $B$ if it is an $\varepsilon$-cone smooth point of $B$ for some $\varepsilon>0$. Moreover, we say that $B$ is cone smooth if each $x \in \partial B$ is a cone smooth point of $B$.

Lemma 2.3. Let $f, g \in S_{X^{*}}, \alpha, \beta, \delta>0$, and $y \in \delta \beta B_{X}$. Suppose that

$$
\alpha+\beta+\alpha \beta<\frac{1}{2} \text { and } \inf g\left(y+\left[\mathrm{C}(\alpha, f) \cap \delta(1+\beta) B_{X}\right]\right) \geq 0 .
$$

Then $\|g-f\| \leq 2(\alpha+\beta+\alpha \beta)$.

Proof. By homogeneity, we can (and do) suppose that $\delta=1$. Put $\gamma=\alpha+$ $\beta+\alpha \beta$. For each $x \in \mathrm{C}(\gamma, f) \cap S_{X}$, we have $\|x-y\| \leq 1+\beta$ and $f(x-y) \geq$ $\gamma-\beta=\alpha(1+\beta) \geq \alpha\|x-y\|$. This shows that $\mathrm{C}(\gamma, f) \cap S_{X}$ is contained in $y+\left[\mathrm{C}(\alpha, f) \cap(1+\beta) B_{X}\right]$. Hence by our assumption, inf $g\left(\mathrm{C}(\gamma, f) \cap S_{X}\right) \geq 0$. Since $\mathrm{C}(\gamma, f)$ is a cone, we obtain that inf $g(\mathrm{C}(\gamma, f))=0$. Now, [2, Lemma 1.1] (based on the "Parallel Hyperplane Lemma") implies that $\|g-f\| \leq 2 \gamma$, which completes the proof.

\section{General Results on tilings}

In the present section, if not specified otherwise, $X$ denotes a real topological vector space (t.v.s., for short).

Given a body $B \subset X$, a point $x \in \partial B$ is called a conical [resp. flat] point of $B$ if the set (in fact, a convex cone) $\bigcup_{t>0} t(B-x)$ is closed [resp. is a closed halfspace]. The body $B$ is plump [resp. flat] if each of its conical [resp. boundary] points is flat.

Let us specify that the terms nonconical point and nonflat point refer only to boundary points of the body. By the way, these two notions are equivalent for plump bodies.

Recall that a hereditarily Baire space is a topological space whose all nonempty closed subspaces are Baire spaces.

Let $x$ be a boundary point of a body $B$. The following simple facts hold. 
$(\alpha) x$ is a flat point of $B$ if and only if there exists a (necessarily unique)

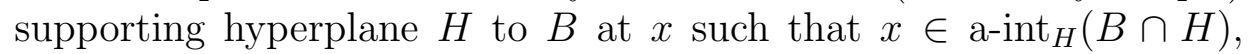
where a-int ${ }_{H}(B \cap H)$ is the relative algebraic interior (or "core") of $B \cap H$ in $H$.

( $\beta)$ If $X$ is hereditarily Baire then in $(\alpha)$ we can write $\operatorname{int}_{H}(B \cap H)$, the topological relative interior of $B \cap H$ in $H$, instead of a-int ${ }_{H}(B \cap H)$.

$(\gamma)$ Hence if $x$ is a flat point of $B$ then $x$ is a smooth point (Fréchet smooth point if $X$ is a Banach space) of $B$.

$(\delta)$ Each smooth conical point is a flat point (see also [11, 1.3]); and, in normed spaces, each LUR point is a nonconical point. Hence if each boundary point of $B$ is either a smooth or a LUR point then $B$ is plump.

As usual, by "arbitrarily near to $x$ " we mean "in any neighborhood of $x$ ". The following lemma is based on $[11,2.1]$.

Lemma 3.1. Let $x$ be a common boundary point of two bodies $B, B^{\prime} \subset X$ whose interiors are disjoint.

(i) If $x$ is a nonconical point of $B$, then arbitrarily near to $x$ there exists a nonflat point of $B$ that does not belong to $B^{\prime}$.

(ii) If $X$ is hereditarily Baire and $x$ is a flat point of both $B, B^{\prime}$, then $x$ belongs to $A:=\operatorname{int}\left(B \cup B^{\prime}\right)$ and all boundary points of $B$ or $B^{\prime}$ that belong to $A$ are flat.

Proof. (i) Let $H$ be a closed hyperplane through $x$ that separates $B$ and $B^{\prime}$. By 2.1 in [11], arbitrarlily near to $x$ there exist nonflat points of $B$ that do not belong to $H$. This shows (i). Part (ii) follows easily from the observation $(\beta)$ above.

A tiling of $X$ is a covering of $X$ by at least two bodies whose interiors are pairwise disjoint. A disjoint tiling is a tiling whose members are pairwise disjoint. A point $x \in X$ is a singular point for a tiling $\mathcal{B}$ if each neighborhood of $x$ meets infinitely many members of $\mathcal{B}$. The points of $\bigcup_{B \in \mathcal{B}} \partial B$ that are not singular are called regular points.

A tiling $\mathcal{B}$ is plump if each of its members is plump. Smooth tiling, Fréchet smooth tiling, LUR tiling, etc. are defined in an analogous way. We say that $\mathcal{B}$ has no common flat points if no point of $X$ is a flat point of two distinct members of $\mathcal{B}$. A trivial tiling has been defined in Introduction.

The following simple fact is well known; we sketch a proof of it for sake of completeness.

Fact 3.2. Each disjoint covering of $\mathbb{R}$ by at least two nonempty closed convex subsets of $\mathbb{R}$ is uncountable. In particular, $\mathbb{R}$ admits no disjoint tiling. 
Proof. Suppose that $\mathcal{B}$ is such a covering and that $\mathcal{B}$ is countable. Then the set $S:=\bigcup_{I \in \mathcal{B}} \partial I$ is a countable closed (nonempty) set. It is easy to see that $S$ has no isolated points. On the other hand, a direct application of the Baire category theorem to the complete metric space $S=\bigcup_{s \in S}\{s\}$ gives existence of an isolated point of $S$. This contradiction completes the proof of the first part. The second part holds by separability.

Lemma 3.3. Let $U \subset X$ be an open convex set, and $B \subset X$ a body whose boundary intersects $U$. Assume that each point of $\partial B \cap U$ is a flat point of $B$. Then each connected component of $\partial B \cap U$ is of the form $H \cap U$ where $H \subset X$ is a closed hyperplane.

Proof. For $x \in \partial B \cap U$, let $H_{x}$ be the unique supporting hyperplane to $B$ at $x$. We claim that $H_{x} \cap U \subset \partial B$. Indeed, if this is not the case, there exists $y \in\left(H_{x} \cap U\right) \backslash B$; then $[x, y] \cap B=[x, z]$ for some $z \in(x, y) \subset H_{x} \cap U$, but this is impossible since clearly $z$ is a non-flat point of $B$.

Assume without any loss of generality that $0 \in \operatorname{int} B$, and let us consider the function $\theta: \partial B \cap U \rightarrow X^{*}$ such that, for $x \in \partial B \cap U, \theta(x)$ is the unique functional of $X^{*}$ such that

$$
[\theta(x)](x)=1=\sup [\theta(x)](B) .
$$

By the claim above, the map $\theta$ is locally constant, and hence it is constant on each connected component of $\partial B \cap U$.

Lemma 3.4. Let $U \subset X$ be an open convex set. Let $E_{n} \subset X, n \in \mathbb{N}$, be closed halfspaces intersecting $U$, such that $E_{n} \cap U \subset E_{n+1} \cap U$ for each $n$. Then either $C:=\bigcup_{n \in \mathbb{N}}\left(E_{n} \cap U\right)$ contains $U$, or the set $\partial C \cap U$ is of the form $H \cap U$ where $H \subset X$ is a closed hyperplane.

Proof. Suppose that $U \backslash C \neq \emptyset$. By our monotonicity assumption, the set $C$ is convex. Notice that also the set $U \backslash C=\bigcap_{n \in \mathbb{N}}\left(U \backslash E_{n}\right)$ is convex. Since $\operatorname{int}(C \cap U) \neq \emptyset$, there exists a closed hyperplane $H \subset X$ that separates $C \cap U$ and $U \backslash C$. Since the union of the latter two sets is $U$ and they belong to opposite halfspaces determined by $H$, either of the two sets must contain the relative interior in $U$ of one of the two opposite halfspaces. This easily implies the assertion.

For simplicity of formulation, if $E, F, U$ are sets and $E \cap U=F \cap U$, we shall say that $E$ coincides with $F$ in $U$.

Theorem 3.5. Let $\mathcal{B}$ be a tiling of a locally convex metrizable t.v.s. $X, B$ a member of $\mathcal{B}$, and $x \in \partial B$ a non-flat point of $B$. Then at least one of the following three conditions is satisfied.

(a) $x$ belongs to at least three different members of $\mathcal{B}$. 
(b) $x$ belongs to two different members $B, B^{\prime}$ of $\mathcal{B}$ and, in some neighborhood of $x, B \cup B^{\prime}$ coincides with a closed halfspace.

(c) Arbitrarily near to $x$ there exist non-flat boundary points of members of $\mathcal{B}$ non containing $x$.

Proof. Assume the contrary, that is, all (a),(b),(c) are false. In particular, $\mathcal{B}_{x}:=\{C \in \mathcal{B}: x \in C\}$ contains at most two elements (one of which is $B$ ), and there exists an open convex neighborhood $U$ of $x$ in which the only non-flat boundary points of members of $\mathcal{B}$ belong to some element of $\mathcal{B}_{x}$.

We claim that arbitrarily near to $x$ there are points of members of $\mathcal{B} \backslash \mathcal{B}_{x}$. This is clear if $\mathcal{B}_{x}=\{B\}$. Let $\mathcal{B}_{x}$ contain also some $B^{\prime} \neq B$. If our claim is false, $B \cup B^{\prime}$ contains a neighborhood $V$ of $x$. But this easily implies that, in such a neighborhood $V$, the set $B$ (and $B^{\prime}$, too) coincides with a closed halfspace, contradicting the assumption that $x$ is a non-flat point of $B$.

Now, observe that if $C \in \mathcal{B} \backslash \mathcal{B}_{x}$ then, by Lemma 3.3, each component of $\partial C \cap U$ coincides in $U$ with a closed hyperplane.

Fix a sequence $\left\{C_{n}\right\} \subset \mathcal{B} \backslash \mathcal{B}_{x}$ such that the distances $d\left(x, C_{n}\right)$ tend decreasingly to 0 . By the above observation, for each $n \in \mathbb{N}$, there exists a closed hyperplane $H_{n}$ such that $H_{n} \cap U \subset \partial C_{n}$, and $H_{n}$ separates $C_{n} \cap U$ and $C_{n+1} \cap U$, as well as $C_{n} \cap U$ and $\left(\cup \mathcal{B}_{x}\right) \cap U$. Let $E_{n}$ be the closed halfspace such that $\partial E_{n}=H_{n}$ and $C_{n} \cap U \subset E_{n}$. Since the sets $E_{n} \cap U$ form an increasing sequence which does not cover $U$, Lemma 3.4 implies that the relative boundary of $\bigcup_{n \in \mathbb{N}}\left(E_{n} \cap U\right)$ in $U$ is of the form $H \cap U$ where $H$ is a closed hyperplane. Clearly, $H$ is a supporting hyperplane to $B$ at $x$, and $\left(\bigcup \mathcal{B}_{x}\right) \cap U$ is contained in one of the two closed halfspaces, say $G$, determined by $H$.

If $\mathcal{B}_{x}=\{B\}$ then, in any neighborhood of $x, B$ does not coincide with $G$, since $x$ is a non-flat point. If $\mathcal{B}_{x}=\left\{B, B^{\prime}\right\}$ (with $B^{\prime} \neq B$ ) then, in any neighborhood of $x, B \cup B^{\prime}$ does not coincide with $G$, since (b) is false. This means that, arbitrarily near to $x$, there exists a boundary point $y$ of some $D \in \mathcal{B} \backslash \mathcal{B}_{x}$ such that $y \in \operatorname{int} G$. Notice that, for each such $D, D \cap U$ is contained in $G$ since it is disjoint from each $C_{n} \cap U(n \in \mathbb{N})$.

Proceeding as above, we can find a sequence $\left\{D_{n}\right\} \subset \mathcal{B} \backslash \mathcal{B}_{x}$ and closed halfspaces $F_{n}, n \in \mathbb{N}$, such that:

- $\partial F_{n} \cap U \subset \partial D_{n}$;

- $\partial F_{n}$ separates $D_{n} \cap U$ and $D_{n+1} \cap U$, as well as $D_{n} \cap U$ and $\left(\bigcup \mathcal{B}_{x}\right) \cap U$;

- $D_{n} \cap U \subset F_{n}$;

- the sequence $\left\{F_{n} \cap U\right\}$ is increasing and contained in $G$.

Now, Lemma 3.4 again implies that the relative boundary of $\bigcup_{n \in \mathbb{N}}\left(F_{n} \cap U\right)$ in $U$ is of the form $H^{\prime} \cap U$ where $H^{\prime}$ is a closed hyperplane through $x$. Moreover, $H^{\prime} \cap U \subset G$. But $x \in \partial G \cap H^{\prime} \cap U$ easily implies that $H^{\prime}=\partial G(=H)$. So we obtain that $B \cap U \subset G$ and also $B \cap U \subset \bigcap_{n \in \mathbb{N}}\left(U \backslash \overline{F_{n}}\right) \subset \overline{X \backslash G}$, 
and consequently $B \cap U \subset H$, which is impossible since $B$ is convex and has nonempty interior. This contradiction completes the proof.

Definition 3.6 ([1]). Let $B$ be a body in a t.v.s. $X$. We say that $x$ is a $Q P$-point of $B$ iff there exists a neighborhood $V$ of $x$ such that $[x, y] \subset \partial B$ whenever $y \in V \cap \partial B$.

We shall need the following simple lemma. (By the way, it is strictly connected with [11, Theorem 5.1] and with the easy implication in the following result of V.P. Fonf [5]: a separable Banach space admits a locally finite tiling by bounded bodies if and only if it is isomorphically polyhedral.)

Lemma 3.7. Let $\mathcal{B}$ be a tiling of a t.v.s. $X$, and let $x \in \bigcup_{B \in \mathcal{B}} \partial B$ be a regular (i.e., non-singular) point for $\mathcal{B}$. Then $x$ is a QP-point of each member of $\mathcal{B}$ that contains $x$. In particular, if $x$ is contained in only two members of $\mathcal{B}$ then $x$ is a flat QP-point for both of them.

Proof. Assume that $x=0$ and denote $\mathcal{B}_{0}=\{B \in \mathcal{B}: 0 \in B\}$. Since $\mathcal{B}_{0}$ is finite, it is easy to see that $0 \in \operatorname{int}\left(\bigcup \mathcal{B}_{0}\right)$ (see also [9, Theorem 1.1]). Let $V \subset X$ be a balanced (i.e., starshaped w.r.t. 0 ) open neighborhood of 0 such that $V \subset \operatorname{int}\left(\bigcup \mathcal{B}_{0}\right)$. Notice that the sets $B \cap V\left(B \in \mathcal{B}_{0}\right)$ form a finite "tiling" of $V$. Now, fix arbitrary $B \in \mathcal{B}$ and $y \in \partial B \cap V$. Then necessarily $y \in B^{\prime}$ for some $B^{\prime} \in \mathcal{B}_{0} \backslash\{B\}$, and hence $[0, y] \subset B \cap B^{\prime} \subset \partial B$. This shows that 0 is a QP-point of $B$. Finally, if $\mathcal{B}_{0}=\left\{B, B^{\prime}\right\}$, let $V$ be as above and let $H$ be a closed hyperplane through $x$ that separates $B$ and $B^{\prime}$. Notice that then necessarily $\partial B \cap V=\partial B^{\prime} \cap V=H \cap V$. The proof is complete.

The following theorem is the main result of the present section. Given a set $E \subset X$ and a neighborhood $V$ of the origin, we write

$$
\operatorname{diam}(E) \prec V
$$

to say that $x-y \in V$ whenever $x, y \in E$. Thus if $X$ is a normed space then $\operatorname{diam}(E) \prec \varepsilon B_{X}$ is the same as $\operatorname{diam}(E) \leq \varepsilon$.

Theorem 3.8. Let $\mathcal{B}$ be a tiling of a locally convex t.v.s. X. Assume that at least one of the following conditions is satisfied:

(i) $\mathcal{B}$ is disjoint;

(ii) $X$ is completely metrizable, $\mathcal{B}$ is plump and nontrivial;

(iii) $X$ is completely metrizable, $\mathcal{B}$ has no common flat points.

Then $\mathcal{B}$ is uncountable. Moreover, if

$$
\mathcal{P}:=\{(x, B): B \in \mathcal{B}, x \in \partial B\}=\bigcup_{n \in \mathbb{N}} \mathcal{P}_{n} \quad \text { where } \quad \mathcal{P}_{1} \subset \mathcal{P}_{2} \subset \ldots,
$$

then there exists $m \in \mathbb{N}$ with the following property: 
(*) for each neighborhood $V$ of 0 there exist three couples $\left(x_{i}, B_{i}\right) \in \mathcal{P}_{m}$, $i=1,2,3$, such that the bodies $B_{i}$ are pairwise distinct and $\operatorname{diam}\left\{x_{1}, x_{2}, x_{3}\right\} \prec V$.

Proof. (i) Let $\mathcal{B}$ be a disjoint tiling of $X$. Let $Y \subset X$ be a straight line intersecting at least two members of $\mathcal{B}$, and let $\mathcal{B}^{\prime}=\{B \cap Y: B \in \mathcal{B}, B \cap Y \neq$ $\emptyset\}$. Since $\mathcal{B}^{\prime}$ is a disjoint cover of $Y$ by nonempty closed convex sets, $\mathcal{B}^{\prime}$ (and hence also $\mathcal{B}$ ) is uncountable by Fact 3.2. Since $Y$ is separable, the family $\mathcal{B}^{\prime \prime}=\left\{B \in \mathcal{B}^{\prime}: \operatorname{int}_{Y} B \neq \emptyset\right\}$ is at most countable. Hence $\mathcal{C}:=\{B \in \mathcal{B}:$ $\#(B \cap Y)=1\}$ is uncountable. For $n \in \mathbb{N}$, let $\mathcal{C}_{n}$ be the collection of all $B \in \mathcal{C}$ such that $B \cap Y=\{x\}$ and $(x, B) \in \mathcal{P}_{n}$. Since $\mathcal{C}=\bigcup_{n} \mathcal{C}_{n}$, there exists $m \in \mathbb{N}$ such that $\mathcal{C}_{m}$ is uncountable. The set $M:=\bigcup_{B \in \mathcal{C}_{m}}(B \cap Y)$ is uncountable, and hence it has an accumulation point $w \in Y$. Then arbitrarily near to $w$ we can find three distinct points $x_{1}, x_{2}, x_{3} \in M$. Let $B_{1}, B_{2}, B_{3} \in \mathcal{C}_{m}$ be such that $x_{i} \in \partial B_{i}(i=1,2,3)$. Then $B_{1}, B_{2}, B_{3}$ are pairwise distinct, $\left(x_{i}, B_{i}\right) \in \mathcal{P}_{m}$ for $i=1,2,3$, and $\operatorname{diam}\left\{x_{1}, x_{2}, x_{3}\right\}$ "can be taken arbitrarily small". Thus (i) is proved.

(ii) Let $X$ be completely metrizable and let $\mathcal{B}$ be a nontrivial plump tiling of $X$. Let us use the following notations.

$$
\begin{aligned}
& \mathcal{B}_{x}:=\{B \in \mathcal{B}: x \in B\} \quad(x \in X), \\
& \eta B:=\{x \in \partial B: x \text { is a nonflat point of } B\} \quad(B \in \mathcal{B}), \\
& \eta \mathcal{B}:=\bigcup_{B \in \mathcal{B}} \eta B, \quad S:=\bigcup_{B \in \mathcal{B}} \partial B .
\end{aligned}
$$

Notice that $S$ is closed in $X$. If $\eta \mathcal{B}=\emptyset$ then each element of $\mathcal{B}$ is flat; in this case, Lemma 3.3 (with $U=X$ ) easily implies that $\mathcal{B}$ is a trivial tiling. Hence we have that $\eta \mathcal{B} \neq \emptyset$, and the closure $\overline{\eta \mathcal{B}}$ is a Baire space.

Suppose that $\mathcal{B}$ is countable. Then $\overline{\eta \mathcal{B}}=\bigcup_{B \in \mathcal{B}}(\overline{\eta \mathcal{B}} \cap \partial B)$, and hence there exist $B_{0} \in \mathcal{B}, x \in \overline{\eta \mathcal{B}} \cap \partial B_{0}$ and a convex open neighborhood $U$ of $x$ such that $\overline{\eta \mathcal{B}} \cap U \subset \partial B_{0}$. Clearly, $x$ cannot be a flat point of $B_{0}$; indeed, otherwise we can suppose that, in $U$, the body $B_{0}$ coincides with a closed halfspace, and then $\overline{\eta \mathcal{B}} \cap U=\emptyset$, which is impossible. Thus $x \in \eta B_{0}$. In this case, one of the conditions (a),(b) in Theorem 3.5 must hold. It is easy to see that this implies existence of some $B_{1} \in \mathcal{B}$ such that $x \in \eta B_{1}$. Notice that $x$ is a nonconical point of $B_{1}$ since $B_{1}$ is plump. By Lemma 3.1(i), there exists a nonflat point of $B_{1}$ contained in $U \backslash B_{0}$. But this contradicts the choice of $x$. We have proved that $\mathcal{B}$ is uncountable.

Now, let us show the second part of (ii). If some $x \in S$ belongs to three distinct $B_{1}, B_{2}, B_{3} \in \mathcal{B}$, there exists $m \in \mathbb{N}$ such that $\left(x, B_{i}\right) \in \mathcal{P}_{m}$ for all $i \in\{1,2,3\}$, and then $(*)$ trivially holds with $x_{1}=x_{2}=x_{3}=x$. 
So, let us suppose that each $x \in S$ belongs to at most two members of $\mathcal{B}$. Then $\overline{\eta \mathcal{B}}=\bigcup_{n \in \mathbb{N}} A_{n}$ where

$$
A_{n}=\left\{x \in \overline{\eta \mathcal{B}}:(x, B) \in \mathcal{P}_{n} \text { whenever } B \in \mathcal{B}_{x}\right\} .
$$

Since $\overline{\eta \mathcal{B}}$ is a Baire space, there is $m \in \mathbb{N}$ such that $\overline{A_{m}}$ has nonempty relative interior in $\overline{\eta \mathcal{B}}$. That is, there exist $x_{1} \in A_{m}$ and a convex open neighborhood $U$ of $x_{1}$ such that $U \cap \overline{\eta \mathcal{B}} \subset \overline{A_{m}}$. By Lemma 3.1(ii), $x_{1}$ cannot be a common flat point of two distinct elements of $\mathcal{B}_{x_{1}}$. Thus either $x_{1}$ is a nonflat point of some $B_{1} \in \mathcal{B}_{x_{1}}$, or $\mathcal{B}_{x_{1}}=\left\{B_{1}\right\}$ and $x_{1}$ is a flat point of $B_{1}$.

Case 1: $x_{1} \in \eta B_{1}$. Since we must have either (b) or (c) in Theorem 3.5, arbitrarily near to $x_{1}$ there exists a nonflat point $x$ of some $B \in \mathcal{B} \backslash\left\{B_{1}\right\}$. If $x \in B_{1}$, we can apply Lemma 3.1(i) to find, arbitrarily near to $x$, a point $x^{\prime} \in \eta B \backslash B_{1}$. So we can always suppose that $x \in U \backslash B_{1}$, and hence $x \in \overline{A_{m}}$. Arbitrarily near to $x$ there exists $x_{2} \in A_{m} \cap\left(U \backslash B_{1}\right)$.

Case 2: $\mathcal{B}_{x_{1}}=\left\{B_{1}\right\}$ and $x_{1} \notin \eta B_{1}$. Since $x_{1} \in \overline{\eta \mathcal{B}}$ and all points sufficiently near to $x_{1}$ are flat for $B_{1}$, arbitrarily near to $x_{1}$ we can find a nonflat point $x$ of some $B \in \mathcal{B} \backslash\left\{B_{1}\right\}$. Proceeding as in Case 1, we get that arbitrarily near to $x$ there exists $x_{2} \in A_{m} \cap\left(U \backslash B_{1}\right)$.

In both Case 1 and 2, fix $B_{2} \in \mathcal{B}_{x_{2}}$. Now, considering an open convex neighborhood $U^{\prime}$ of $x_{2}$ such that $U^{\prime} \subset U \backslash B_{1}$, we can proceed as above to find, arbitrarily near to $x_{2}$, a point $x_{3} \in A_{m} \cap\left(U^{\prime} \backslash B_{2}\right)$. Fix $B_{3} \in \mathcal{B}_{x_{3}}$ and notice that the bodies $B_{1}, B_{2}, B_{3}$ are pairwise distinct, $\left(x_{i}, B_{i}\right) \in \mathcal{P}_{m}$ for $i=1,2,3$, and $\operatorname{diam}\left\{x_{1}, x_{2}, x_{3}\right\}$ "can be taken arbitrarily small". This completes the proof of part (ii).

(iii) Let $X$ be completely metrizable and let $\mathcal{B}$ be a tiling that has no common flat points. We shall use the above notations (2). Assume that $\mathcal{B}$ is countable. Then $S=\bigcup_{B \in \mathcal{B}} \partial B$ is a Baire space (since it is closed), and hence there exists $B_{0} \in \mathcal{B}$ for which $\partial B_{0}$ has nonempty relative interior in $S$. That is, there exist $x \in \partial B_{0}$ and a convex open neighborhood $U$ of $x$ such that $U \cap S \subset \partial B_{0}$. We can (and do) suppose that $U \cap \partial B_{0}$ is connected, and so $U \backslash \partial B_{0}$ has two components. Hence there exists $B_{1} \in \mathcal{B} \backslash\left\{B_{0}\right\}$ such that $U \subset B_{0} \cup B_{1}$. It follows that $U \cap \partial B_{0}=U \cap \partial B_{1}$ is contained in a closed hyperplane and hence $x$ is a common flat point of $B_{0}$ and $B_{1}$. This contradiction shows that $\mathcal{B}$ must be uncountable.

Now, as in (ii), if some $x \in S$ belongs to three distinct members of $\mathcal{B}$, we are immediately done. So, let us assume that $\# \mathcal{B}_{x} \leq 2$ for each $x \in S$. For $n \in \mathbb{N}$, denote $A_{n}=\left\{x \in S:(x, B) \in \mathcal{P}_{n}\right.$ whenever $\left.B \in \mathcal{B}_{x}\right\}$, and notice that $S=\bigcup_{n \in \mathbb{N}} A_{n}$. Hence, for some $m \in \mathbb{N}, \overline{A_{m}}$ has nonempty relative interior in $S$, that is, there exist $x_{1} \in A_{m}$ and a convex open neighborhood $U$ of $x_{1}$ such that $U \cap S \subset \overline{A_{m}}$. We claim that $x_{1}$ is necessarily a singular point of $\mathcal{B}$. Indeed, if not, we can apply Lemma 3.7 to obtain that $\mathcal{B}_{x_{1}}=\left\{B_{0}, B_{1}\right\}$ with $B_{0} \neq B_{1}$, and $x_{1}$ is a common flat point of $B_{0}, B_{1}$, which is impossible. 
Thus $x_{1}$ is a singular point of $\mathcal{B}$. Fix $B_{1} \in \mathcal{B}_{x_{1}}$. Then arbitrarily near to $x_{1}$ there exists $x \in \partial B \cap U$ with $B \in \mathcal{B} \backslash\left\{B_{1}\right\}$; and arbitrarily near to $x$ there exists $x_{2} \in A_{m} \backslash B_{1}$. Fix $B_{2} \in \mathcal{B}_{x_{2}}$ and notice that $B_{2} \neq B_{1}$. Now, arbitrarily near to $x_{1}$ find $x^{\prime} \in \partial B^{\prime}$ with $x^{\prime} \in \mathcal{B} \backslash\left\{B_{1}, B_{2}\right\}$; and arbitrarily near to $x^{\prime}$ find $x_{3} \in A_{m} \backslash\left(B_{1} \cup B_{2}\right)$. Fix $B_{3} \in \mathcal{B}_{x_{3}}$. Then $B_{1}, B_{2}, B_{3}$ are pairwise distinct, $\left(x_{i}, B_{i}\right) \in \mathcal{P}_{m}$ for $i=1,2,3$, and $\operatorname{diam}\left\{x_{1}, x_{2}, x_{3}\right\}$ "can be taken arbitrarily small". This proves (iii), and we are done.

Corollary 3.9 (cf. [11]). Let $\mathcal{B}$ be a countable plump tiling of a locally convex completely metrizable t.v.s. Then $\mathcal{B}$ is trivial.

\section{Applications to normed and Banach spaces}

In what follows, $X$ is a normed space.

\subsection{Smoothness-type prohibitive conditions.}

For the definition of $\varepsilon-C S(B)$ see Section 2.

Lemma 4.1. There exists a constant $H \in(0,1)$ such that if $\varepsilon>0, B_{1}, B_{2}, B_{3}$ are convex bodies in $X$ with pairwise disjoint interiors and $x_{i} \in \varepsilon-\operatorname{CS}\left(B_{i}\right)$ $(i=1,2,3)$, then $\operatorname{diam}\left(\left\{x_{1}, x_{2}, x_{3}\right\}\right)>H \varepsilon$.

Proof. We claim that our lemma holds with $H=1 / 51$. Put $\alpha=1 / 7$ and $\beta=1 / 50$, and notice that

$$
\gamma:=\alpha+\beta+\alpha \beta=\frac{29}{175}<\frac{1}{6}
$$

By homogeneity, we can (and do) suppose that $\varepsilon=1+\beta=51 / 50$. Then $H \varepsilon=1 / 50$.

Now, assume that the assertion is false, i.e., $\operatorname{diam}\left(\left\{x_{1}, x_{2}, x_{3}\right\}\right) \leq H \varepsilon=\beta$. Let $f_{i} \in S_{X^{*}}(i=1,2,3)$ be such that

$$
K_{i}:=x_{i}-\left[\mathrm{C}\left(\alpha, f_{i}\right) \cap(1+\beta) B_{X}\right] \subset B_{i} .
$$

Since the bodies $K_{1}, K_{2}, K_{3}$ have pairwise disjoint interiors, for each $i, j \in$ $\{1,2,3\}, i<j$, there exists $g_{i j} \in S_{X^{*}}$ such that $\sup g_{i j}\left(-K_{j}\right) \leq \inf g_{i j}\left(-K_{i}\right)$, that is,

$\inf g_{i j}\left(\left(x_{j}-x_{i}\right)+\left[\mathrm{C}\left(\alpha, f_{i}\right) \cap(1+\beta) B_{X}\right]\right) \geq \sup g_{i j}\left(\left[\mathrm{C}\left(\alpha, f_{j}\right) \cap(1+\beta) B_{X}\right]\right) \geq 0$.

By Lemma 2.3, $\left\|g_{i j}-f_{i}\right\| \leq 2 \gamma$. In a similar way we obtain $\left\|g_{i j}+f_{j}\right\| \leq 2 \gamma$. But now, since $2 f_{1}=\left(f_{1}-g_{12}\right)+\left(f_{2}+g_{12}\right)-\left(f_{2}-g_{23}\right)-\left(f_{3}+g_{23}\right)+\left(f_{3}+g_{13}\right)+\left(f_{1}-g_{13}\right)$, we obtain $2=\left\|2 f_{1}\right\| \leq 6 \cdot 2 \gamma<2$, a contradiction.

Theorem 4.2. Let $\mathcal{B}$ be a tiling of a normed space $X$. Assume that at least one of the following conditions is satisfied:

(i) $\mathcal{B}$ is disjoint;

(ii) $X$ is complete, $\mathcal{B}$ is plump and nontrivial;

(iii) $X$ is complete, $\mathcal{B}$ has no common flat points. 
Then $\mathcal{B}$ is not cone smooth.

Proof. Let us suppose on the contrary that $\mathcal{B}$ is cone smooth. Let $\mathcal{P}$ be as in Theorem 3.8 and, for each $n \in \mathbb{N}$, let

$$
\mathcal{P}_{n}:=\{(x, B): B \in \mathcal{B}, x \in(1 / n)-\operatorname{CS}(B)\} .
$$

Then $\mathcal{P}_{1} \subset \mathcal{P}_{2} \subset \ldots$, moreover, since $\mathcal{B}$ is cone smooth, $\mathcal{P}=\bigcup_{n} \mathcal{P}_{n}$.

Now, let $H$ be the constant from Lemma 4.1. By Theorem 3.8, there exist $m \in \mathbb{N}$ and three couples $\left(x_{i}, B_{i}\right) \in \mathcal{P}_{m}, i=1,2,3$, such that the bodies $B_{i}$ are pairwise distinct and $\operatorname{diam}\left\{x_{1}, x_{2}, x_{3}\right\}<H / m$. But this contradicts Lemma 4.1.

Now, since Fréchet smooth bodies are cone smooth (see Section 2) and plump (see $(\delta)$ in Section 3 ), we immediately obtain the following

Corollary 4.3. Let $\mathcal{B}$ be a Fréchet smooth tiling of a Banach space $X$. Then $\mathcal{B}$ is trivial. In particular, $X$ does not admit any Fréchet smooth tiling by bounded bodies.

\subsection{Rotundity-type prohibitive conditions for ball tilings.}

We shall need the following elementary observation. As usual, if $c, y \in X$ are two distinct points then $(c, y)$ denotes the relatively open line segment with endpoints $c, y$, that is, $(c, y)=\{(1-t) c+t y: 0<t<1\}$.

Observation 4.4. Given $B=B(c, r), y \in \partial B$, and $0<\rho<r$, there exists $B^{\prime}=B\left(c^{\prime}, \rho\right)$ such that $c^{\prime} \in(c, y), B^{\prime} \subset B$ and $y \in \partial B^{\prime}$.

Lemma 4.5. Let $X$ be a normed space, $\alpha>0, \varepsilon \geq 0$, and $B_{0}, B_{1}, B_{2} \subset X$ three balls of radius at least $\alpha$, whose interiors are pairwise disjoint. Consider three points $y_{i} \in \partial B_{i}, i=0,1,2$, and denote $x_{0}=\frac{y_{0}-c_{0}}{\left\|y_{0}-c_{0}\right\|}$ where $c_{0}$ is the center of $B_{0}$. If $\operatorname{diam}\left\{y_{0}, y_{1}, y_{2}\right\} \leq \alpha \varepsilon$ then

$$
\operatorname{diam}\left\{y \in S_{X}:\left\|x_{0}+y\right\| \geq 2-\varepsilon\right\} \geq 2-2 \varepsilon .
$$

Proof. By homogeneity and by Observation 4.4, we can assume that $\alpha=1$ and the three balls have unit radius and that $\operatorname{diam}\left\{y_{0}, y_{1}, y_{2}\right\} \leq \varepsilon$. We can also assume that $c_{0}=0$, so that $x_{0}=y_{0}$. For $i=1,2$, let $c_{i}$ be the center of $B_{i}$, and denote $z_{i}=c_{i}-x_{0}$ and $\bar{z}_{i}=\frac{z_{i}}{\left\|z_{i}\right\|}$. Since $z_{i}=\left(c_{i}-y_{i}\right)+\left(y_{i}-y_{0}\right)$ and $\left\|c_{i}-y_{i}\right\|=1$, we have $1-\varepsilon \leq\left\|z_{i}\right\| \leq 1+\varepsilon$, and hence $\left\|\bar{z}_{i}-z_{i}\right\| \leq \varepsilon$. Since $x_{0}+\bar{z}_{i}=\left(c_{i}-c_{0}\right)+$ $\left(\bar{z}_{i}-z_{i}\right)$ and $\left\|c_{i}-c_{0}\right\| \geq 2$, we have $\left\|\frac{x_{0}+\bar{z}_{i}}{2}\right\| \geq 1-\frac{\varepsilon}{2}$, and hence the points $\bar{z}_{1}, \bar{z}_{2}$ belong to the set from (3). Finally, since $\bar{z}_{1}-\bar{z}_{2}=\left(c_{1}-c_{2}\right)+\left(\bar{z}_{1}-z_{1}\right)+\left(z_{2}-\bar{z}_{2}\right)$, we obtain that $\left\|\bar{z}_{1}-\bar{z}_{2}\right\| \geq\left\|c_{1}-c_{2}\right\|+\left\|\bar{z}_{1}-z_{1}\right\|+\left\|z_{2}-\bar{z}_{2}\right\| \geq 2-2 \varepsilon$, completing the proof.

Definition 4.6. We shall say that $x \in S_{X}$ is a locally non-D2 (or LND2) point of $B_{X}$ if there exists $\delta>0$ such that

$$
\operatorname{diam}\left\{y \in S_{X}:\left\|\frac{x+y}{2}\right\| \geq 1-\delta\right\}<2 .
$$


In the above definition, "D2" stands for "diameter 2". Notice that each LUR point of $B_{X}$ is clearly a LND2 point.

Theorem 4.7. Let $\mathcal{B}$ be a tiling by balls of a normed space $X$. Assume that at least one of the following conditions is satisfied:

(i) $\mathcal{B}$ is disjoint;

(ii) $X$ is a Banach space and $\mathcal{B}$ is plump;

(iii) $X$ is a Banach space and $\mathcal{B}$ has no common flat points.

Then there exists $x \in S_{X}$ such that $x$ is neither a cone smooth point nor a $L N D 2$ point of $B_{X}$.

The proof is similar to the proof of Theorem 4.2.

Proof. Let us suppose on the contrary that this is not the case, that is, each $x \in S_{X}$ is either a cone smooth point or a LND2 point of $B_{X}$. If $B \in \mathcal{B}$ let us denote by $r_{B}$ and $c_{B}$ the radius and the center of $B$, respectively. Let $\mathcal{P}$ be as in Theorem 3.8 and, for each $n \in \mathbb{N}$, let $\mathcal{P}_{n} \subset \mathcal{P}$ be the set of all couples $(x, B)$ such that $r_{B} \geq 1 / n$ and at least one of the following two conditions holds:

(a) $\operatorname{diam}\left\{y \in S_{X}:\left\|\frac{x-c_{B}}{\left\|x-c_{B}\right\|}+y\right\| \geq 2-\frac{1}{n}\right\}<2-\frac{2}{n}$;

(b) $x \in \frac{1}{n}-\mathrm{CS}(B)$.

Clearly $\mathcal{P}_{1} \subset \mathcal{P}_{2} \subset \ldots$, and $\mathcal{P}=\bigcup_{n} \mathcal{P}_{n}$.

Let $H$ be the constant from Lemma 4.1. By Theorem 3.8, there exists $m \in \mathbb{N}$ and three couples $\left(x_{i}, B_{i}\right) \in \mathcal{P}_{m}, i=1,2,3$, such that $\operatorname{diam}\left\{x_{1}, x_{2}, x_{3}\right\}<H / m^{2}$ and the balls $B_{i}(i=1,2,3)$ are pairwise distinct and with radii at least $1 / \mathrm{m}$. Now, it sufficies to consider the following two cases.

Case 1: $x_{i} \in \frac{1}{m}-\mathrm{CS}\left(B_{i}\right)$ for each $i=1,2,3$. This case is impossible by Lemma 4.1 since $\operatorname{diam}\left\{x_{1}, x_{2}, x_{3}\right\}<H / m^{2} \leq H / m$.

Case 2: $\operatorname{diam}\left\{y \in S_{X}:\left\|\frac{x_{1}-c_{B_{1}}}{\left\|x_{1}-c_{B_{1}}\right\|}+y\right\| \geq 2-\frac{1}{m}\right\}<2-\frac{2}{m}$. Since

$$
\operatorname{diam}\left\{x_{1}, x_{2}, x_{3}\right\}<H / m^{2}<1 / m^{2},
$$

Lemma 4.5 (with $\alpha=\frac{1}{m}$ ) gives that $\operatorname{diam}\left\{y \in S_{X}:\left\|\frac{x_{1}-c_{B_{1}}}{\left\|x_{1}-c_{B_{1}}\right\|}+y\right\| \geq 2-\frac{1}{m}\right\} \geq$ $2-\frac{2}{m}$, which is a contradiction.

Since each LUR point is a LND2 point, and each Fréchet smooth point is a cone smooth point, we obtain the following corollary.

Corollary 4.8. Let $\mathcal{B}$ be a tiling by balls of a normed space $X$. Assume that either $\mathcal{B}$ is disjoint or $X$ is complete. Then there exists $x \in S_{X}$ such that $x$ is neither a Fréchet smooth point nor a LUR point of $B_{X}$. In particular, a LUR Banach space admits no tiling by balls, and a normed LUR space admits no disjoint tiling by balls. 


\subsection{One-point prohibitive conditions for large-ball tilings.}

Now we are going to consider tilings by balls whose radii are bounded away from 0. Let us recall two well known geometrical notions. Given a norm-one functional $f \in X^{*}$, a slice of $B_{X}$, determined by $f$, is any set of the form

$$
S(f, \delta)=\left\{y \in B_{X}: f(y)>1-\delta\right\} \quad \text { where } \delta \in(0,1) .
$$

A point $x \in S_{X}$ is said to be a strongly exposed point of $B_{X}$ if there exists a norm-one $f \in X^{*}$ such that $f(x)=1$ and $\operatorname{diam}(S(f, \delta)) \rightarrow 0$ as $\delta \rightarrow 0^{+}$.

Theorem 4.9. Let $X$ be a normed space, suppose that at least one of the following conditions holds:

(a) there exists $x \in S_{X}$ such that $X$ is LND2 at $x$, and $x$ is not a QP-point for $B_{X}$;

(b) there exists a LUR point in $S_{X}$;

(c) there exists a Fréchet smooth point $x \in S_{X}$, which is not a QP-point for $B_{X}$, and the unique norm-one functional $f_{x} \in X^{*}$ that supports $B_{X}$ at $x$ determines a slice $\Sigma$ of $B_{X}$ with $\operatorname{diam}(\Sigma)<2$;

(d) there exists a Fréchet smooth point $x \in S_{X}$ which is a strongly exposed point of $B_{X}$.

Then $X$ admits no tiling by balls with radii bounded away from 0 .

Proof. Let $\mathcal{B}$ be a tiling of $X$ by balls with radii bounded away from 0 . We can (and do) suppose that $B_{X} \in \mathcal{B}$.

First assume (a). Let $x \in S_{X}$ be such that $X$ is LND2 at $x$ and such that $x$ is not a QP-point for $B_{X}$. By Lemma 3.7, $x$ is a singular point for $\mathcal{B}$. Thus there exist a sequence $\left\{B_{n}\right\}$ of pairwise distinct elements of $\mathcal{B} \backslash\left\{B_{X}\right\}$, and points $y_{n} \in \partial B_{n}$ such that $y_{n} \rightarrow x$. Fix an arbitrary $\delta>0$. Since $\operatorname{diam}\left\{x, y_{n}, y_{n+1}\right\} \rightarrow 0$, we can apply Lemma 4.5 to deduce that, for each $\varepsilon \in(0,2 \delta)$, we have

$\operatorname{diam}\left\{y \in S_{X}:\|x+y\| \geq 2-2 \delta\right\} \geq \operatorname{diam}\left\{y \in S_{X}:\|x+y\| \geq 2-\varepsilon\right\} \geq 2-2 \varepsilon$.

It follows that $\operatorname{diam}\left\{y \in S_{X}:\left\|\frac{x+y}{2}\right\| \geq 1-\delta\right\}=2$ for each $\delta>0$. But this contradicts the fact that $x$ is a LND2 point for $B_{X}$, and part (a) is proved. Moreover, since each LUR point $x \in S_{X}$ satisfies (a), part (b) immediately follows.

Now assume (c). By the first part of the proof of part (a), $\operatorname{diam}\left\{y \in S_{X}\right.$ : $\left.\left\|\frac{x+y}{2}\right\| \geq 1-\delta\right\}=2$ for each $\delta>0$. This easily implies existence of a sequence $\left\{y_{n}\right\} \subset S_{X}$ such that $\left\|\frac{x+y_{n}}{2}\right\| \rightarrow 1$, and $\operatorname{diam}\left(\left\{y_{n}\right\}_{n \geq n_{0}}\right)=2$ for each $n_{0} \in \mathbb{N}$. By convexity of the norm, for each $n \in \mathbb{N}$ there exists $z_{n} \in\left(x, y_{n}\right)$ such that $\left\|z_{n}\right\|=\min \left\{\|z\|: z \in\left[x, y_{n}\right]\right\}$. It is not difficult to see that

$$
\left\|z_{n}\right\| \geq\left\|x+y_{n}\right\|-1
$$


(indeed, if $z_{n}^{\prime} \in\left(x, y_{n}\right)$ is such that $\frac{z_{n}+z_{n}^{\prime}}{2}=\frac{x+y_{n}}{2}$, then $\left\|x+y_{n}\right\|=\left\|z_{n}+z_{n}^{\prime}\right\| \leq$ $\left.\left\|z_{n}\right\|+1\right)$. For each $n \in \mathbb{N}$, let $f_{n} \in X^{*}$ be a norm-one functional that separates $\left\|z_{n}\right\| B_{X}$ and $\left[x, y_{n}\right]$; clearly,

$$
f_{n}\left(z_{n}\right)=\left\|z_{n}\right\|=f_{n}(x)=f_{n}\left(y_{n}\right) .
$$

Notice that $\left\|z_{n}\right\| \rightarrow 1$, that is, $f_{n}(x) \rightarrow 1$. Since $x$ is a Fréchet smooth point of $B_{X}$, we have that $f_{n} \rightarrow f_{x}$ in the norm topology (see, e.g., [4, Corollary 7.22]). It follows that $f_{x}\left(y_{n}\right) \rightarrow 1$. In particular, $y_{n}$ belongs to $\Sigma$ for each sufficiently large $n$, and hence $\operatorname{diam}(\Sigma) \geq 2$. This contradicition proves (c). Finally, part (d) follows easily from part (c). Our theorem is proved.

\section{A LUR Disjoint tiling of $\ell_{1}(\Gamma)$}

In this section we show that, for some suitable uncountable set $\Gamma$, the Banach space $\ell_{1}(\Gamma)$ admits a disjoint tiling by bounded LUR bodies. Our construction is based on a construction by $\mathrm{V}$. Klee [8] of a disjoint tiling of $\ell_{1}(\Gamma)$ by translates of the unit ball. Let us start with some preliminary work.

Let $\Gamma$ be a nonempty set and let us denote by $\|\cdot\|_{i}$ the canonical norm of $\ell_{i}(\Gamma)(i=1,2)$. For $x \in \ell_{1}(\Gamma)$, let us define $\|x\|=\left(\|x\|_{1}^{2}+\|x\|_{2}^{2}\right)^{1 / 2}$. It is known that $\|\cdot\|$ is an equivalent LUR norm on $\ell_{1}(\Gamma)([4$, Lemma 13.26]).

In what follows, let $M>4 \sqrt{2}$ be a fixed constant. For $\gamma \in \Gamma$ and $x \in \ell_{1}(\Gamma)$, let us define

$$
\begin{aligned}
\|x\|_{1}^{\gamma} & =\sum_{\beta \in \Gamma \backslash\{\gamma\}}|x(\beta)|+\frac{1}{M}|x(\gamma)| \\
\|x\|_{2}^{\gamma} & =\left(\sum_{\beta \in \Gamma \backslash\{\gamma\}}|x(\beta)|^{2}+\frac{1}{M^{2}}|x(\gamma)|^{2}\right)^{1 / 2} \\
\|x\|^{\gamma} & =\left[\left(\|x\|_{1}^{\gamma}\right)^{2}+\left(\|x\|_{2}^{\gamma}\right)^{2}\right]^{1 / 2}
\end{aligned}
$$

It is easy to see that $\left(\ell_{1}(\Gamma),\|\cdot\|^{\gamma}\right)$ and $\left(\ell_{1}(\Gamma),\|\cdot\|\right)$ are linearly isometric, and hence $\|\cdot\|^{\gamma}$ is an equivalent LUR norm on $\ell_{1}(\Gamma)$.

As usual, $B_{\|\cdot\|}$ and $S_{\|\cdot\|}$ denote the closed unit ball and the unit sphere of a norm $\|\cdot\|$. Notice that the polar set $\left(B_{\|\cdot\|}\right)^{\circ}$ is the corresponding dual unit ball. We shall need the following fact, the easy proof of which is left to the reader.

Fact 5.1. Let $x \in S_{\|\cdot\|^{\gamma}}$, and $f \in \ell_{\infty}(\Gamma)$. Assume that $f=a_{1} f_{1}+a_{2} f_{2}$, where $f_{i} \in\left(B_{\|\cdot\|_{i}^{\gamma}}\right)^{\circ}(i=1,2)$, and $a_{1}, a_{2} \in \mathbb{R}$ are such that $a_{1}^{2}+a_{2}^{2} \leq 1$. Then:

(a) $f \in\left(B_{\|\cdot\| \gamma}\right)^{\circ}$;

(b) if $a_{i}=f_{i}(x)=\|x\|_{i}^{\gamma}(i=1,2)$ then $f(x)=1=\sup f\left(B_{\|\cdot\|^{\gamma}}\right)$. 
Let $\left\{e_{\beta}\right\}_{\beta \in \Gamma}$ denote the canonical basis of $\ell_{1}(\Gamma)$. Let us define

$$
z_{\gamma}:=(M / \sqrt{2}) e_{\gamma} \quad(\gamma \in \Gamma)
$$

It is elementary to see that

$$
\left\|z_{\gamma}\right\|_{1}^{\gamma}=\left\|z_{\gamma}\right\|_{2}^{\gamma}=1 / \sqrt{2} \text { and }\left\|z_{\gamma}\right\|^{\gamma}=1 \quad(\gamma \in \Gamma) .
$$

Lemma 5.2. Let $\gamma_{0}$ and $\gamma_{1}$ be two distinct elements of $\Gamma$. Let $x \in S_{\|\cdot\| \gamma_{0}}$ be such that $x\left(\gamma_{1}\right)=0$. Then there exists $f \in \ell_{\infty}(\Gamma) \backslash\{0\}$ such that $f(x)=$ $\sup f\left(B_{\|\cdot\| \gamma_{0}}\right)$ and $f\left(z_{\gamma_{1}}\right)=\sup f\left(B_{\|\cdot\| \gamma^{\gamma_{1}}}\right)$.

Proof. Let us define $f_{1}, f_{2}, f \in \ell_{\infty}(\Gamma)$ as follows.

$$
\begin{aligned}
f_{1}(\gamma) & = \begin{cases}\operatorname{sign}[x(\gamma)] / M & \text { if } \gamma=\gamma_{0}, \\
1 & \text { if } \gamma=\gamma_{1}, \\
\operatorname{sign}[x(\gamma)] & \text { if } \gamma \neq \gamma_{0}, \gamma_{1} ;\end{cases} \\
f_{2}(\gamma) & = \begin{cases}x(\gamma) /\left[M^{2}\|x\|_{2}^{\gamma_{0}}\right] & \text { if } \gamma=\gamma_{0}, \\
0 & \text { if } \gamma=\gamma_{1}, \\
x(\gamma) /\|x\|_{2}^{\gamma_{0}} & \text { if } \gamma \neq \gamma_{0}, \gamma_{1} ;\end{cases} \\
f & =\|x\|_{1}^{\gamma_{0}} f_{1}+\|x\|_{2}^{\gamma_{0}} f_{2} .
\end{aligned}
$$

An easy calculation shows that:

- $f_{1} \in\left(B_{\|\cdot\|_{1}^{\gamma_{0}}}\right)^{\circ}$ and $f_{1}(x)=\|x\|_{1}^{\gamma_{0}}$;

- $f_{2} \in\left(B_{\|\cdot\|_{2}^{\gamma_{0}}}\right)^{\circ}$ and $f_{2}(x)=\|x\|_{2}^{\gamma_{0}}$;

- $f(x)=1=\sup f\left(B_{\|\cdot\| \gamma_{0}}\right)$ by Fact 5.1.

It remains to show that $\sup f\left(B_{\|\cdot\|^{\gamma_{1}}}\right)=f\left(z_{\gamma_{1}}\right)$. Let us define $g_{1}, g_{2}, g \in \ell_{\infty}(\Gamma)$ as follows.

$$
\begin{aligned}
g_{1}(\gamma) & = \begin{cases}1 / M & \text { if } \gamma=\gamma_{1}, \\
2 f(\gamma) /\left[\|x\|_{1}^{\gamma_{0}} M\right] & \text { if } \gamma \neq \gamma_{1}\end{cases} \\
g_{2}(\gamma) & = \begin{cases}1 / M & \text { if } \gamma=\gamma_{1}, \\
0 & \text { if } \gamma \neq \gamma_{1}\end{cases} \\
g & =\left\|z_{\gamma_{1}}\right\|_{1}^{\gamma_{1}} g_{1}+\left\|z_{\gamma_{1}}\right\|_{2}^{\gamma_{1}} g_{2}=(1 / \sqrt{2})\left(g_{1}+g_{2}\right) .
\end{aligned}
$$

Since $\|\cdot\|_{1}^{\gamma_{0}} \geq\|\cdot\|_{2}^{\gamma_{0}}$, we easily obtain $\|\cdot\|_{1}^{\gamma_{0}} \geq\|\cdot\|^{\gamma_{0}} / \sqrt{2}$. Moreover, since $|f(\gamma)| \leq 2(\gamma \in \Gamma)$, we have $\frac{2|f(\gamma)|}{\|x\|_{1}^{\gamma 0} M} \leq \frac{2 \sqrt{2}}{M}|f(\gamma)| \leq \frac{4 \sqrt{2}}{M} \leq 1$. This easily implies that $g_{1} \in\left(B_{\|\cdot\|_{1}^{\gamma_{1}}}\right)^{\circ}$.

It is also easy to verify that $g_{2} \in\left(B_{\|\cdot\|_{2}^{\gamma_{1}}}\right)^{\circ}$, and $g_{i}\left(z_{\gamma_{1}}\right)=1 / \sqrt{2}=\left\|z_{\gamma_{1}}\right\|_{i}^{\gamma_{1}}$ $(i=1,2)$. Using Fact 5.1, we obtain

$$
g\left(z_{\gamma_{1}}\right)=1=\sup g\left(B_{\|\cdot\| \gamma_{1}}\right) .
$$


To finish the proof, notice that $f\left(\gamma_{1}\right)=\|x\|_{1}^{\gamma_{0}}$ and $g\left(\gamma_{1}\right)=\frac{\sqrt{2}}{M}$. Now it is easy to verify that $f=\frac{M}{\sqrt{2}}\|x\|_{1}^{\gamma_{0}} g$, and hence $f\left(z_{\gamma_{1}}\right)=\sup f\left(B_{\|\cdot\| \gamma_{1}}\right)$.

Lemma 5.3. Let $\gamma_{0}$ and $\gamma_{1}$ be two distinct elements of $\Gamma$. Let $x_{\gamma_{1}}$ be an element of $\ell_{1}(\Gamma) \backslash B_{\|\cdot\| \gamma_{0}}$ such that $x_{\gamma_{1}}\left(\gamma_{1}\right)=0$. Then the sets $B_{0}:=B_{\|\cdot\| \gamma_{0}}$ and $B_{1}:=x_{\gamma_{1}}+z_{\gamma_{1}}+B_{\|\cdot\| \gamma_{1}}$ are disjoint.

Proof. Since $x_{\gamma_{1}}\left(\gamma_{1}\right)=0$, by Lemma 5.2, there exists $f \in \ell_{\infty}(\Gamma) \backslash\{0\}$ such that

$$
f\left(z_{\gamma_{1}}\right)=\sup f\left(B_{\|\cdot\| \gamma_{1}}\right)=-\inf f\left(B_{\|\cdot\| \gamma_{1}}\right) \quad \text { and } \quad \sup f\left(B_{0}\right)=f\left(\frac{x_{\gamma_{1}}}{\left\|x_{\gamma_{1}}\right\|^{\gamma_{0}}}\right) .
$$

Since $\left\|x_{\gamma_{1}}\right\|^{\gamma_{0}}>1$ and clearly $f\left(x_{\gamma_{1}}\right)>0$, we obtain

$$
\sup f\left(B_{0}\right)=\inf f\left(\frac{x_{\gamma_{1}}}{\left\|x_{\gamma_{1}}\right\|^{\gamma_{0}}}+z_{\gamma_{1}}+B_{\|\cdot\| \gamma_{1}}\right)<\inf f\left(B_{1}\right)
$$

which proves that $B_{0}$ and $B_{1}$ are disjoint.

Let us recall that $\Gamma$ is a regular cardinal if $\Gamma$ is a cardinal such that each cofinal subset of $\Gamma$ has the same cardinality as $\Gamma$. (A set $A \subset \Gamma$ is called cofinal with $\Gamma$ if each element of $\Gamma$ is less or equal to some element of $A$.) It is known that there exist arbitrarily large infinite regular cardinals $\Gamma$ such that $\Gamma^{\aleph_{0}}=\Gamma$ (see [8]); such cardinals are clearly uncountable. Given a set $M$, we denote by $|M|$ its cardinality.

Theorem 5.4. Let I be a cardinal such that $\mathrm{I}^{\aleph_{0}}=\mathrm{I}$. Then $\ell_{1}(\mathrm{I})$ admits a disjoint tiling $\mathcal{C}$ by centrally symmetric bounded LUR bodies.

Proof. Let us proceed in two steps.

STEP1. Let $\Gamma$ be a regular cardinal such that $\Gamma^{\aleph_{0}}=\Gamma$. Put $X:=\ell_{1}(\Gamma)$. We shall show that $X$ admits a disjoint tiling $\mathcal{B}$ by centrally symmetric bounded LUR bodies.

Proceeding as in the proof of [8, Theorem 1.2], it is not difficult to show that if $\mathrm{K}$ is an infinite cardinal then $\left|\ell_{1}(\mathrm{~K})\right|=\mathrm{K}^{\aleph_{0}}$. Then $|X|=\Gamma$ and hence we may assume that $X$ is well-ordered by an antireflexive relation $\prec$ as to be order-isomorphic with $\Gamma$.

For $\beta \in \Gamma$, denote $X_{\beta}=\{x \in X: x(\gamma)=0 \forall \gamma \geq \beta\}$. Let us inductively construct families $\mathcal{B}_{\beta}(\beta \in \Gamma)$. For $\alpha=\min \Gamma$, we define

$$
x_{\alpha}=0, \quad y_{\alpha}=z_{\alpha}, \quad \mathcal{B}_{\alpha}:=\left\{y_{\alpha}+B_{\|\cdot\|^{\alpha}}\right\}
$$

(recall that $z_{\alpha}=M e_{\alpha} / \sqrt{2}$ ).

Now, let $\beta \in \Gamma \backslash\{\alpha\}$, and assume that the families $\mathcal{B}_{\gamma}$ have been already defined for all $\gamma<\beta$. Put $Y_{\beta}=\bigcup_{\gamma<\beta}\left(\bigcup \mathcal{B}_{\gamma}\right)$.

(i) If $X_{\beta} \subset Y_{\beta}$, define $\mathcal{B}_{\beta}=\bigcup_{\gamma<\beta} \mathcal{B}_{\gamma}$; 
(ii) if else, let $x_{\beta}$ be the first point of $X_{\beta} \backslash Y_{\beta}$, and define

$$
\mathcal{B}_{\beta}=\left(\bigcup_{\gamma<\beta} \mathcal{B}_{\gamma}\right) \cup\left\{y_{\beta}+B_{\|\cdot\|^{\beta}}\right\} \quad \text { where } \quad y_{\beta}=x_{\beta}+z_{\beta} .
$$

We shall show that the family $\mathcal{B}=\bigcup_{\beta \in \Gamma} \mathcal{B}_{\beta}$ has the desired properties.

First of all, let us show that the elements of $\mathcal{B}$ are pairwise disjoint. Indeed, let $B_{0}=y_{\beta_{0}}+B_{\|\cdot\|^{\beta_{0}}}$ and $B_{1}=y_{\beta_{1}}+B_{\|\cdot\|^{\beta_{1}}}$ be two distinct elements of $\mathcal{B}$ (with $\beta_{0}, \beta_{1} \in \Gamma$ ). By our construction, we may suppose that $\beta_{0}<\beta_{1}, x_{\beta_{1}} \notin$ $y_{\beta_{0}}+B_{\|\cdot\| \beta_{0}}$ and $x_{\beta_{1}}\left(\beta_{1}\right)=0$. Since $x_{\beta_{1}}\left(\beta_{1}\right)=x_{\beta_{0}}\left(\beta_{1}\right)=z_{\beta_{0}}\left(\beta_{1}\right)=0$ and $x_{\beta_{1}}-x_{\beta_{0}}-z_{\beta_{0}} \notin B_{\|\cdot\|^{\beta_{0}}}$, we can apply Lemma 5.3 to conclude that $B_{\|\cdot\|^{\beta_{0}}}$ and $\left(x_{\beta_{1}}-x_{\beta_{0}}-z_{\beta_{0}}\right)+B_{\|\cdot\|_{1}}$ are disjoint. It follows that $B_{1}$ and $B_{2}$ are disjoint.

It remains to show that $\mathcal{B}$ covers $X$. Suppose that this is not the case and let $w$ be the first element of $X \backslash \bigcup \mathcal{B}$. Put $L=\{x \in X: x \prec w\}$. Since $L$ is covered by $\mathcal{B}$, for each $v \in L$ there exists $\beta_{v} \in \Gamma$ such that $v \in y_{\beta_{v}}+B_{\|\cdot\|^{\beta}}$. Let us consider the set $A \subset \Gamma$ defined by

$$
A:=\operatorname{supp}(w) \cup \bigcup_{v \in L}\left[\operatorname{supp}(v) \cup \operatorname{supp}\left(y_{\beta_{v}}\right)\right]
$$

(here $\operatorname{supp}(u)$ denotes the support of $u \in X)$. Notice that

$$
|A| \leq(2|L|+1) \cdot \aleph_{0}=\max \left\{|L|, \aleph_{0}\right\}<\Gamma .
$$

Since $\Gamma$ is regular and $|A|<\Gamma, A$ is not cofinal in $\Gamma$. Thus there exists $\delta \in \Gamma$ such that $\beta<\delta$ for each $\beta \in A$. Clearly, $w \in X_{\delta} \backslash(\cup \mathcal{B}) \subset X_{\delta} \backslash Y_{\delta}$.

We claim that $w$ is the first element of $X_{\delta} \backslash Y_{\delta}$. Indeed, if $v \in L$, then $\beta_{v} \in A$ and $\beta_{v}<\delta$, which implies that $v \in y_{\beta_{v}}+B_{\|\cdot\|_{v}} \subset \bigcup \mathcal{B}_{\beta_{v}} \subset Y_{\delta}$.

But this means that $w=x_{\delta} \in\left(y_{\delta}+B_{\|\cdot\|^{\delta}}\right) \in \mathcal{B}$, which is a contradiction that concludes the proof of the first step.

STEP2. To prove the general case, we proceed as in the proof of $[9$, Theorem 3.2]. Given our cardinal I, consider $\Gamma:=\left(2^{\mathrm{I}}\right)^{+}$, the successor of the cardinal $2^{\mathrm{I}}$. By $\left[8\right.$, Remark 1.1], $\Gamma$ is regular and $\Gamma^{\aleph_{0}}=\Gamma$. By Step $1, \ell_{1}(\Gamma)$ admits a disjoint tiling $\mathcal{B}$ by centrally symmetric bounded LUR bodies. For each $B \in \mathcal{B}$ let $c_{B}$ be its center of symmetry.

Let $\mathrm{I}_{0}=\mathrm{I} \subset \Gamma$ and $\mathcal{C}_{0}=\emptyset$. Let us inductively construct $\mathrm{I}_{\beta} \subset \Gamma$ and $\mathcal{C}_{\beta} \subset \mathcal{B}$ for $\beta<\omega_{1}$. Assume that $\mathrm{I}_{\gamma}$ and $\mathcal{C}_{\gamma}$ have been already defined for all $\gamma<\beta$. If $\beta$ is a limit ordinal, define $\mathcal{C}_{\beta}=\bigcup_{\gamma<\beta} \mathcal{C}_{\gamma}$ and $\mathrm{I}_{\beta}=\bigcup_{\gamma<\beta} \mathrm{I}_{\gamma}$. If $\beta=\alpha+1$, define

$$
\mathcal{C}_{\beta}=\left\{B \in \mathcal{B} ; B \cap \ell_{1}\left(\mathrm{I}_{\alpha}\right) \neq \emptyset\right\}, \quad \mathrm{I}_{\beta}=\mathrm{I}_{\alpha} \cup \bigcup_{B \in \mathcal{C}_{\beta}} \operatorname{supp}\left(c_{B}\right)
$$

We claim that $\left|\mathrm{I}_{\beta}\right|=\mathrm{I}$ and $\left|\mathcal{C}_{\beta}\right| \leq \mathrm{I}$ for each $\beta<\omega_{1}$. Let us prove our claim by induction. Clearly it holds for $\beta=0$. Now, assume that $\left|\mathrm{I}_{\alpha}\right|=\mathrm{I}=\mathrm{I}^{\aleph_{0}}$ and $\left|\mathcal{C}_{\alpha}\right| \leq \mathrm{I}$ hold for each $\alpha<\beta$. If $\beta$ is a limit ordinal then we have $\mathrm{I} \leq\left|\mathrm{I}_{\beta}\right| \leq$ 
$\aleph_{0} \mathrm{I}=\mathrm{I}$ and $\left|\mathcal{C}_{\beta}\right| \leq \aleph_{0} \mathrm{I}=\mathrm{I}$. For $\beta=\alpha+1$, we have $\left|\mathcal{C}_{\beta}\right| \leq\left|\ell_{1}\left(\mathrm{I}_{\alpha}\right)\right|=\left|\mathrm{I}_{\alpha}\right|^{\aleph_{0}}=\mathrm{I}$, and hence also $\left|\mathrm{I}_{\beta} \backslash \mathrm{I}_{\alpha}\right| \leq \aleph_{0}\left|\mathcal{C}_{\beta}\right| \leq \mathrm{I}$ which implies that $\left|\mathrm{I}_{\beta}\right|=\mathrm{I}$. The claim is proved.

Now, define $\mathrm{J}=\bigcup_{\beta<\omega_{1}} \mathrm{I}_{\beta}$. Clearly, $|\mathrm{J}| \leq \aleph_{1} \mathrm{I}=\mathrm{I}$ and hence $|\mathrm{J}|=\mathrm{I}$. Let $\mathcal{C}^{\prime}=\bigcup_{\beta<\omega_{1}} \mathcal{C}_{\beta}$ and $\mathcal{C}=\left\{B \cap \ell_{1}(\mathrm{~J}): B \in \mathcal{C}^{\prime}\right\}$. Then $\mathcal{C}$ is a disjoint tiling of $\ell_{1}(\mathrm{~J})$ by centrally symmetric bounded LUR bodies. We are done since $\ell_{1}(\mathrm{~J})$ and $\ell_{1}(\mathrm{I})$ are isometrically isomorphic.

Remark 5.5. (a) Let us remark that, under the generalized continuum hypothesis, our assumption $\mathrm{I}^{\aleph_{0}}=\mathrm{I}$ holds for every uncountable cardinal I.

To see this, first suppose that $\mathrm{I}=\mathrm{K}^{+}$(the successor of a cardinal $\mathrm{K}$ ). Then $\mathrm{I}=2^{\mathrm{K}}$ and hence $\mathrm{I}^{\aleph_{0}}=2^{\mathrm{K} \cdot \aleph_{0}}=2^{\mathrm{K}}=\mathrm{I}$. Now, assume that $\mathrm{I}$ is not a successor cardinal; hence $2^{\mathrm{K}}<\mathrm{I}$ whenever $\mathrm{K}<\mathrm{I}$. As usual, we can identify any ordinal $\mathrm{K}$ with the smallest ordinal of cardinality $\mathrm{K}$, and hence also with the interval $[0, K)$ of ordinals. Since I is uncountable, every sequence in $[0, I)$ is contained in $[0, K)$ for some infinite cardinal $\mathrm{K}<\mathrm{I}$. Hence

$$
\mathrm{I} \leq \mathrm{I}^{\aleph_{0}} \leq \sum_{\omega_{0} \leq \mathrm{K}<\mathrm{I}} \mathrm{K}^{\aleph_{0}} \leq \sum_{\omega_{0} \leq \mathrm{K}<\mathrm{I}}\left(2^{\mathrm{K}}\right)^{\aleph_{0}}=\sum_{\omega_{0} \leq \mathrm{K}<\mathrm{I}} 2^{\mathrm{K}} \leq \mathrm{I} \cdot \mathrm{I}=\mathrm{I}
$$

and we are done.

(b) On the other hand, it follows by [8, Proposition 3.5] that if $\ell_{1}\left(\aleph_{1}\right)$ admits a disjoint tiling then we have $\aleph_{1}=2^{\aleph_{0}}$, the continuum hypothesis.

\section{REFERENCES}

[1] D. Amir and F. Deutsch, Suns, moons, and quasi-polyhedra, J. Approx. Theory 6 (1972), 176-201.

[2] C.A. De Bernardi and L. Veselý, On support points and support functionals of convex sets, Israel J. Math. 171 (2009), 15-27.

[3] R. Engelking, General Topology, Second edition, Sigma Series in Pure Mathematics, 6, Heldermann Verlag, Berlin, 1989.

[4] M. Fabian, P. Habala, P. Hájek, V. Montesinos and V. Zizler, Banach Space Theory. The basis for linear and nonlinear analysis, CMS Books in Mathematics/Ouvrages de Mathématiques de la SMC, Springer, New York, 2011.

[5] V.P. Fonf, Three characterizations of polyhedral Banach spaces (Russian), Ukrain. Mat. Zh. 42 (1990), 1286-1290; translation in Ukrainian Math. J. 42 (1990), 11451148 (1991).

[6] V.P. Fonf, A. Pezzotta and C. Zanco, Tiling infinite-dimensional normed spaces, Bull. London Math. Soc. 29 (1997), 713-719.

[7] V.P. Fonf and C. Zanco, Covering a Banach space, Proc. Amer. Math. Soc. 134 (2006), 2607-2611.

[8] V.L. Klee, Dispersed Chebyshev sets and coverings by balls, Math. Ann. 257 (1981), 251-260.

[9] V.L. Klee, Do infinite-dimensional Banach spaces admit nice tilings?, Studia Sci. Math. Hungar. 21 (1986), 415-427. 
[10] V.L. Klee, E. Maluta and C. Zanco, Tiling with smooth and rotund tiles, Fund. Math. 126 (1986), 269-290.

[11] V.L. Klee and C. Tricot, Locally countable plump tilings are flat, Math. Ann. 277 (1987), 315-325.

[12] A. Marchese and C. Zanco, On a question by Corson about point-finite coverings, Israel J. Math. 189 (2012), 55-63.

[13] D. Preiss, Tilings of Hilbert spaces, Mathematika 56 (2010), 217-230.

Dipartimento di Matematica, Università degli Studi, Via C. Saldini 50, 20133 Milano, ItALy

E-mail address: carloalberto.debernardi@gmail.com, libor.vesely@unimi.it 